\title{
Viewpoint Independent Human Motion Analysis in Man-made Environments*
}

\author{
G. Rogez ${ }^{1}$, J.J. Guerrero ${ }^{2}$, J. Martínez ${ }^{1}$ and C. Orrite ${ }^{1}$ \\ ${ }^{1}$ Computer Vision Lab - ${ }^{2}$ Robotics, Perception and Real Time Group \\ I3A - University of Zaragoza, Maria de Luna 1, 50018 Zaragoza, SPAIN \\ \{grogez, jguerrer, jesmar, corrite\}@unizar.es
}

\begin{abstract}
This work addresses the problem of human motion analysis in video sequences of a scene observed by a single fixed camera with high perspective effect. The goal of this work is to make a 2D-Model (made of Shape and Stick figure) viewpoint-insensitive and preprocess the input image for removing the perspective effect. We focus our methodology on using the 3D principal directions of man-made environments and also the direction of motion to transform both 2D-Model and input images to a common frontal view (parallel or orthogonal to the direction of motion) before the fitting process. The inverse transformation is then performed on the resulting human features obtaining a segmented silhouette and a pose estimation in the original input image. Preliminary results are very promising since the proposed algorithm is able to locate head and feet with a better precision than previous one.
\end{abstract}

\section{Introduction}

Recently, the number of cameras deployed for surveillance in urban environments has increased considerably because of their falling cost. The constant human monitoring of these cameras is not feasible because of the required cost. For this reason, human motion analysis has become an active research area in computer vision, since automatic video understanding and activity recognition would enable a single operator to monitor many cameras. The most efficient systems are based on the use of 2D or 3D-models. In this paper we only consider the case of 2D-models that are easier to use and can better handle clutter, partial occlusions and multiple people tracking [11]. The main disadvantage of 2D-models is their restriction to the viewpoint.

Roof-top cameras are widely utilized for video surveillance applications. They usually are located at a significant angle from the floor, most of the time different from the angle of views of typical training set [7] (see Fig.1). Moreover, perspective effects can deform the human silhouette in a way that traditional 2D-models can not be applied correctly. Solving these problems of viewpoint dependency and perspective deformations appears as an urgent requirement for further practical applications.

*Work supported by TIC2003-08382-C05-05 from Spanish Minist. of Sciences and Technology. G. Rogez and J. Martinez are funded under FPU and FPI grants (AP2003-2257 and BES-2004-3741) from Spanish Ministry of Education. 


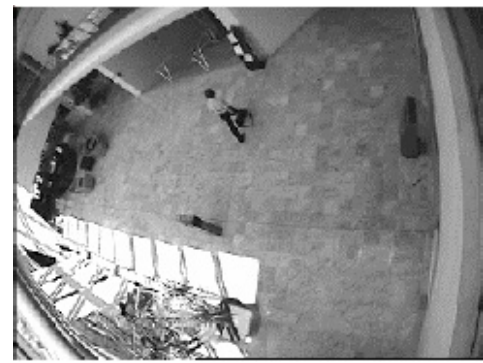

(a)

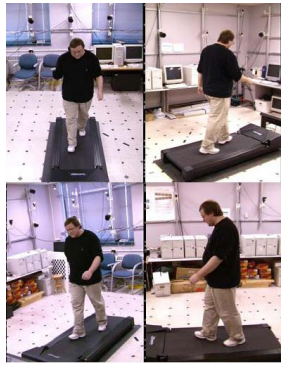

(b)

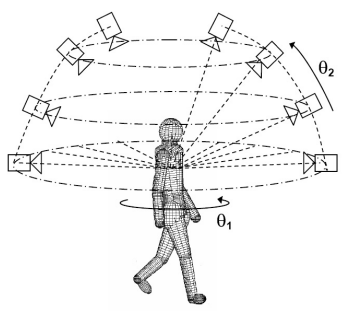

(c)

Figure 1: Video-surveillance (a) and Modo database (b) images. Camera positions and gaze directions (c).

The goal of this work is to investigate how 2D models could be made independent of the point of view from which the scene is observed and how the input image could be pre-processed for applying them in a correct way.

Related Work. The concept of "virtual camera" is introduced in [13] for estimating 3D body pose from multiple views: a mapping is made which is invariant to translation, rotation and scaling of the input. Grauman [6] presented a method for inferring a 3D shape from a single input silhouette with unknown camera viewpoints. The model is learnt by collecting multi-view silhouette examples from calibrated camera rigs and the visual hull inference consists in finding the shape hypotheses most likely to have generated the observed 2D contour. In [3], the viewpoint invariance is achieved by projecting all the images onto the ground plane. In [9] a method is proposed for view invariant gait recognition: considering a person walking along a straight line (making a constant angle with the image plane), a side-view is synthesized by homography.

Contribution. Our goal is to create a 2D Point Distribution Model (PDM) using only few training views and try fitting it to all the possible images obtained by a single calibrated camera with perspective effect. In a previous work [12] the potential of this tool was demonstrated, however the accuracy of the result depends strongly on the similarity of the input image viewpoint with a viewpoint of the training set. Fig.1.c shows the possible positions of a camera with respect to the person observed in the scene. The variation with respect to the vertical axis (along $\theta_{1}$ ) will be compensated directly by the model, using images taken from various views (lateral, diagonal, frontal, etc.) while the variation along the elevation angle, $\theta_{2}$, will be compensated using the dominant $3 \mathrm{D}$ directions of the scene (both training and input images), supposing the person walks in a structured man-made environment full of easy-to-detect straight lines (walls, etc.).

The basic idea is to take advantage of floor and walls information for finding viewpoint independency: the horizontal vanishing line and the vertical vanishing point are used to transform both model and input images to a frontal view $\left(\theta_{2}=0\right)$. In more practical terms, most of times people walks on a planar ground with a vertical posture. These two observations are the basic hypotheses considered in our proposal. The direction of motion is also extracted to define the canonical views, parallel or orthogonal to this direction.

Organization of the paper. The rest of this paper is structured as follows: in Sect. 2, we introduce the algebraic representation. In Sect. 3, we present the model construction and fitting. In Sect. 4, we explain the overall process of human motion analysis. Results are presented in Sect. 5 and conclusions drawn in Sect. 6. 


\section{Correcting the perspective effect}

A vanishing point is the intersection of the projections in the image of a set of parallel world lines. Any set of parallel lines on a plane define a vanishing point and the union of all these vanishing points is the vanishing line of that plane [5]. On Fig.2a, we can see a practical case of ground (horizontal) vanishing line localization.

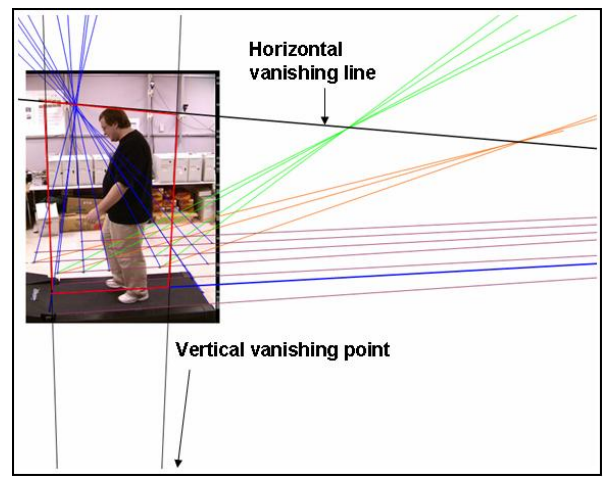

(a)

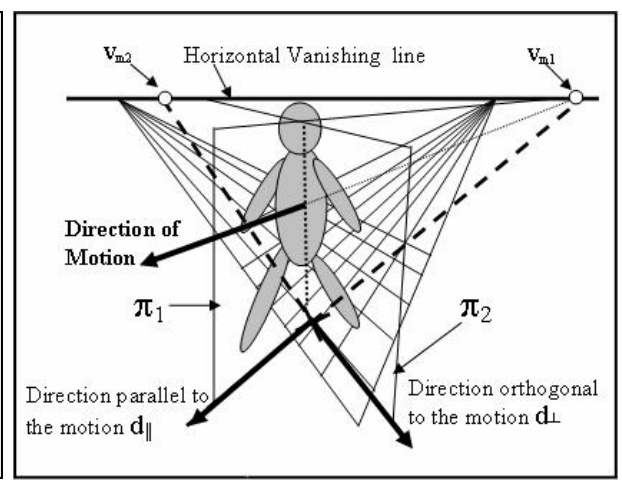

(b)

Figure 2: (a) Vertical vanishing point and horizontal vanishing line estimation in a Mobo's image. (b) Horizontal vanishing point localization thanks to direction of motion (or orthogonal) and ground vanishing line.

Following the classical notation of 3D geometry, points on the image plane, $\mathbf{x}$, are related to points of the $3 \mathrm{D}$ world, $\mathbf{X}$, via a $3 \times 4$ projection matrix $\mathbf{M}$ as:

$$
\mathbf{x}=\mathbf{M X}
$$

where $\mathbf{x}$ and $\mathbf{X}$ are homogeneous vectors and "=" means equality up to scale. As shown in [5], the projective transformation matrix $\mathbf{M}$ can be defined in function of the vanishing points of the dominant 3D directions.

Two main cases have to be considered here: people walking in a direction parallel to the image plane or people walking in a direction globally parallel to the optical axis (towards or backwards the camera). In the first case, we want to transform the image to a canonical view, called "fronto-parallel view", defined as the vertical plane $\left(\pi_{1}\right.$ in Fig. 2b) parallel to the direction of the motion. In that sense, (1) becomes: $\mathbf{x}=\mathbf{H X}$, where $\mathbf{x}=\{x, y, 1\}^{\top}$ and $\mathbf{X}=\left\{X_{m}, Z, 1\right\}^{\top}$ with $\mathbf{X}_{\mathrm{m}}$ the coordinate in the direction of motion. The homography matrix $\mathbf{H}$ is defined as:

$$
\mathbf{H}=\left[\begin{array}{lll}
\mathbf{v}_{\mathrm{m}} & \alpha \mathbf{v}_{Z} & \mathbf{o}
\end{array}\right],
$$

where $\mathbf{v}_{\mathrm{m}}$ is the intersection point (called $\mathbf{v}_{\mathrm{m} 1}$ in Fig.2b) between the direction $\mathbf{d}_{\|}$parallel to the motion and the horizontal vanishing line, $\mathbf{v}_{Z}$ is the vertical vanishing point, $\mathbf{o}$ is the origin of the world coordinate system and $\alpha$ is a scale factor.

In a second case, we project the image to another canonical view, called "frontoorthogonal view", defined as the vertical plane ( $\pi_{2}$ in Fig. 2 ) orthogonal to the direction of the motion. There, $\mathbf{v}_{\mathrm{m}}$ from (2) is the intersection point (called $\mathbf{v}_{\mathrm{m} 2}$ in Fig.2) between the direction orthogonal to the motion $\mathbf{d}_{\perp}$ and the horizon line (horizontal vanishing line). 
In both cases, the transformation matrix $\mathbf{P}$ that projects a point of the image $\mathbf{x}$ onto the $\left(\mathrm{X}_{\mathrm{m}}, \mathrm{Z}\right)$ real world plane as $\mathbf{X}=\mathbf{P x}$ is given by: $\mathbf{P}=\mathbf{H}^{-1}=\left[\mathbf{v}_{\mathrm{m}} \alpha \mathbf{v}_{Z} \mathbf{o}\right]^{-1}$, where the origin $\mathbf{o}$ is translated to the centroid of the considered person. In Sect.4, we will explain how to obtain this factor $\alpha$ and the vanishing point $\mathbf{v}_{\mathrm{m}}$.

\section{Point Distribution Model: Construction and Fitting}

For the training stage, we used the CMU Motion of Body (MoBo) database [7]. Good training shapes were extracted manually from pedestrian silhouettes, considering two walking speeds and 4 different views (frontal, lateral, diagonal and rear-diagonal as shown in Fig.1.b), and trying to get accurate and detailed approximations of the contour (Shape vector). Simultaneously, we labelled 13 fundamental points corresponding to a 2D-Stick model (Skeleton vector). By this process we generated a training database encompassing 10800 Shape-Skeleton vectors, called SS-vectors (2700 vectors for each different view).

\subsection{Database transformation}

The proposed transformations are now applied to the Mobo images (see Fig.3) and to our training database of SS-vectors. The typical "dwarf effect" due to perspective and observed in the frontal view (big head and short legs in the image) is corrected: the image appears distorted but the global figure recovers real morphological proportions. It can also be observed how the transformation tends to place the feet at the same vertical position and remove the perspective effect for the rear-diagonal and diagonal views.

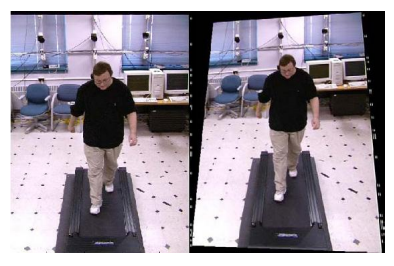

(a)

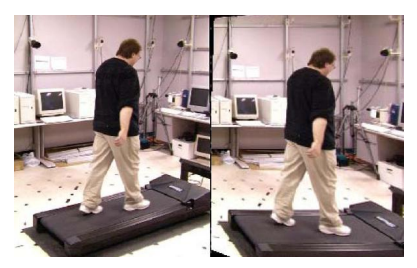

(b)

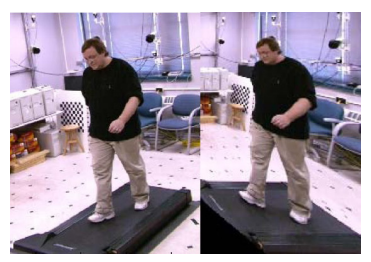

(c)

Figure 3: Original and transformed images for frontal (a), "rear-diagonal" (b) and diagonal (c) training views.

Two sets of models will be generated: two "classical" ones (frontal and lateral view) constructed with the original SS-vectors and two "transformed" ones (fronto-orthogonal and fronto-parallel) constructed with the transformed data. This will allow a comparative of the method proposed in this paper with the classical one. Frontal and diagonal training views data are used for the fronto-orthogonal model construction, while diagonal, lateral and rear-diagonal views data for the fronto-parallel one (see Fig.4). Mixing various views with few angle's variation we want to interpolate SS-vectors absent from the training set.

\subsection{Construction of the Gaussian Mixture Models}

The models are constructed following the same procedure as described in [12]. After the normalization step, our training set of SS-vectors is aligned using Procrustes analysis to free the model of position, size and rotation. As Bowden [2] proposed, first we compute 
linear Principal Component Analysis (PCA) and project all the vectors on PCA basis. Then we carry out a cluster analysis on projections and select an optimum number of clusters (Fig.4a). Each data point is assigned to a cluster, and separate local PCAs are performed independently on each cluster, resulting in the identification of the local model's modes of variation inside each Gaussian distribution of the mixture model (Fig.4b). Parameters related to those modes are used to help the model fit the final data set, by allowing model variability only along the principal variation modes.

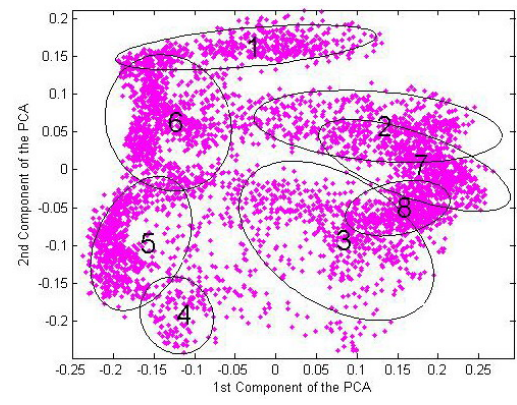

(a)
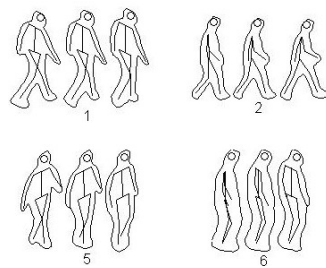

(b)

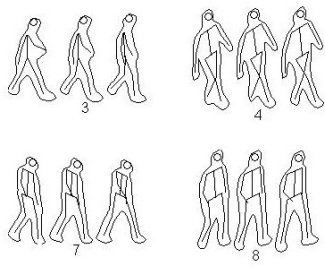

Figure 4: Gaussian mixture model projected on the two first components of the PCA (a) and first mode of variation of the 8 local models of the mixture in the "fronto-parallel" case.

\subsection{Model Fitting and Body Pose Inferring}

Assuming we have an initial estimate for shape and skeleton parameters and an input human blob provided by a background subtraction algorithm, the fitting process (similar to the one proposed in [12]) follows these steps:

1. A shape vector $\mathbf{S}$ is extracted from the blob, looking along straight lines through each model point, following the methodology presented in [4].

2. $\mathbf{S}$ and the skeleton vector $\mathbf{K}$ ( $\overline{\mathbf{K}}$ is used if there is no estimate) are concatenated in $\mathbf{V}=\left[\begin{array}{ll}\mathbf{S}^{\top} & \mathbf{K}^{\top}\end{array}\right]^{\top}$ and projected into the PCA space, obtaining vector $\mathbf{X}$.

3. Find the nearest cluster.

4. Update the parameters to best fit the "local model" defined by its mean $\overline{\mathbf{X}}$, eigenvectors $\Phi$ and eigenvalues $\lambda_{\mathbf{i}}$, obtaining $\mathbf{X}^{\prime}$ [4].

5. We project the new vector $\mathbf{X}^{\prime}$ back to the feature space obtaining $\mathbf{V}^{\prime}$ which contains a new estimation of both contour $\mathbf{S}^{\prime}$ and skeleton $\mathbf{K}^{\prime}: \mathbf{V}^{\prime}=\left[\mathbf{S}^{\prime \top} \mathbf{K}^{\prime \top}\right]^{\top}$.

6. A new background subtraction is applied reducing the threshold value inside the contour defined by $\mathbf{S}^{\prime}$, leading to a better silhouette (See Fig.6 g, h, i).

7. Repeat until convergence and store the data (trajectory, states etc.).

The process can be repeated several times for improving the contour extracted from the blob. This leads to an accurate silhouette segmentation and posture estimation directly obtained from the mapping created between contours and stick figures. 


\section{Human Motion Analysis Process}

\subsection{Camera and Scene Calibration}

In this paper, the transformation matrices are calculated online using the vanishing points evaluated in an off-line stage: the positions of the vanishing points and lines are directly obtained after a manual selection of the parallel lines present in the image (ground and walls). This method makes sense only for man-made environments because of the presence of numerous easy-to-detect straight lines. Previous works for vanishing points detection [10] could be used to automate the process. Using surveillance cameras with a high field of view, a previous lens calibration is usually required to correct the optical distortion.

Given at least four corresponding points, the homography that relates image coordinates and coordinates on the ground plane, can be recovered up to scale. During the scene calibration two homography matrices are calculated from ground data: the one that characterizes the mapping between the image and the ground plane $\mathbf{H}_{\mathrm{g}}$ and another one $\mathbf{H}_{\mathrm{h}}$ that relations the image with a horizontal plane at height $h=1,80$ meters (typical height of an adult person). Given the positions of the feet and/or the head of a "typical person" in the image, these homography matrices can evaluate the ground position of this person and vice versa. They will be used for the evaluation of the scale factor and the determination of the direction of motion.

Once we have calibrated the camera in the scene, the camera cannot be moved, which is a limitation of the proposal. In practice, the orientation of the camera could change, for example, due to the lack of stability of the camera support. A little change in orientation has a great influence in the image coordinates, and therefore invalidates previous calibration. However, if the camera is not changed in position, or position change is small with respect to the depth of the observed scene, the homography could be re-calibrated automatically. An automatic method to compute homographies and line matching between image pairs like presented in [8] can be used, but at the moment it has not been included in our system.

\subsection{Scale factor and horizontal vanishing point}

A ground plane tracking is applied, evaluating the ground position and the motion direction of the tracked person on the ground plane, at each time step. The direction orthogonal to the motion is easily computed on the ground plane. These two orthogonal directions are then projected back to the input image by means of $\mathbf{H}_{\mathrm{g}}$, leading to the direct localization of $\mathbf{v}_{\mathrm{m}}$ and the computation of $\mathbf{P}$.

Scaling parameter $\alpha$ is evaluated considering 3 points: two points on the ground, the ground position and a point localized on the direction of motion (or on the direction orthogonal to the motion) at a given distance, and the corresponding "head position" (position in the horizontal plane at height $h=1,80$ meters). Since the position of these points in real world are known, the height-width ratio can be computed and $\alpha$ can be evaluated so that this ratio still presents the same value in the warped image. 


\subsection{Algorithm}

The algorithm proposed in this paper is valid for every 2D model but we applied it with the PDM presented before. Suppose we have a sequence with a pedestrian walking. We do not take into account the initialization, neither the cases of stationary person, and let it for future work. The detailed block diagram is presented in Fig.5, while Fig.6 shows the intermediate results corresponding to the processing of a particular frame.

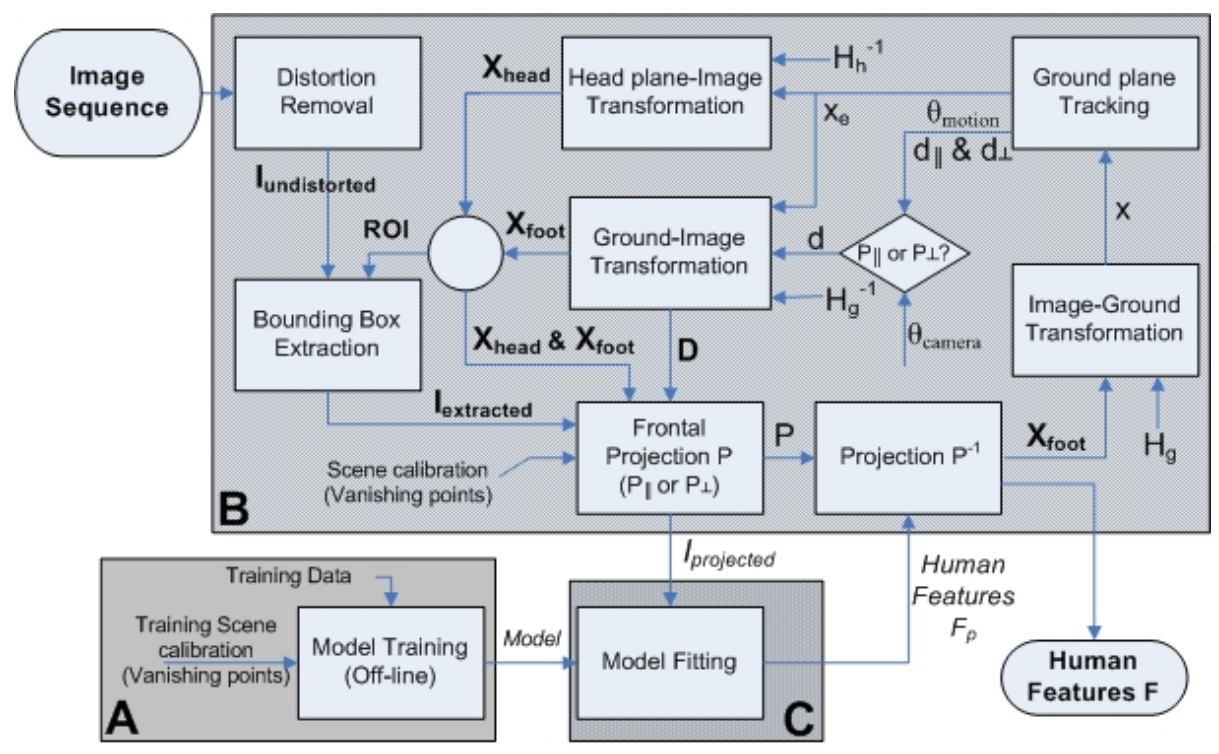

Figure 5: Block diagram of the proposed system.

Firstly, the ground plane tracking provides an estimation of the ground position $\mathrm{x}_{\mathrm{e}}$ that gives directly $\mathbf{X}_{\text {head }}$ and $\mathbf{X}_{\mathbf{f o o t}}$, head and foot positions in the image. Secondly, the tracking also provides an estimation of $\mathbf{d}_{\perp}$ and $\mathbf{d}_{\|}$in the ground plane (direction parallel and orthogonal to the motion). Depending on the orientation with respect to the camera ( $\theta_{\text {motion }}$ and $\theta_{\text {camera }}$ correspond to the angle of motion and the position of the camera in the ground plane), fronto-parallel or fronto-orthogonal transformation is chosen: $\mathbf{D}$ corresponds to the selected direction $\mathrm{d}$ in the image.

$\mathbf{X}_{\text {head }}$ and $\mathbf{X}_{\text {foot }}$ lead to the extraction of a Region of Interest in the image: $\mathbf{I}_{\text {extracted }}$. The Projection $\mathbf{P}$ is then computed and applied on $\mathbf{I}_{\mathbf{e x t r a c t e d}}$, obtaining $I_{\text {projected }}$ that will be used to fit the model. Finally, Human Features are extracted and back-projected in the original image plane, providing a new measure for the ground position $\mathrm{x}$.

\section{Overall Performances and Results}

For the evaluation of the models, a series of sequences of interest are selected from the Caviar project database [1]: in these sequences people are walking in various directions and the changing perspective effect can be noted. The 3 walk sequences available Walkl, Walk 2 and Walk 3 are chosen because of the nature of our "pedestrian" model: the model would not be able to analyze other kinds of motion. 
Since tracking is not the main topic of this paper, we decide to use a manual labelling of the feet in the image, providing directly a reliable ground plane position to the tracking algorithm (a Kalman Filter in this case). The fitting is carried out with both "frontal" and "classical" models and the manual labelling is used for numerical evaluation.

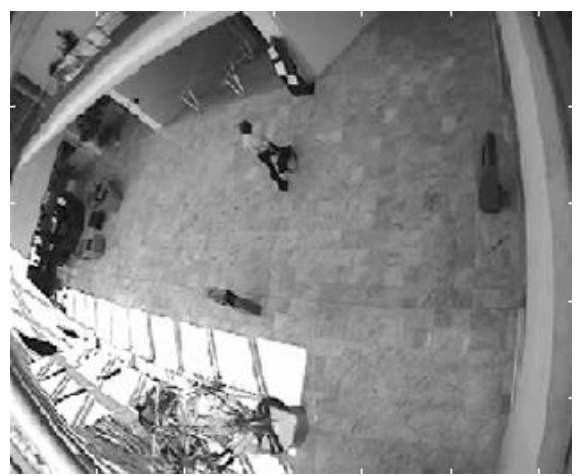

(a)

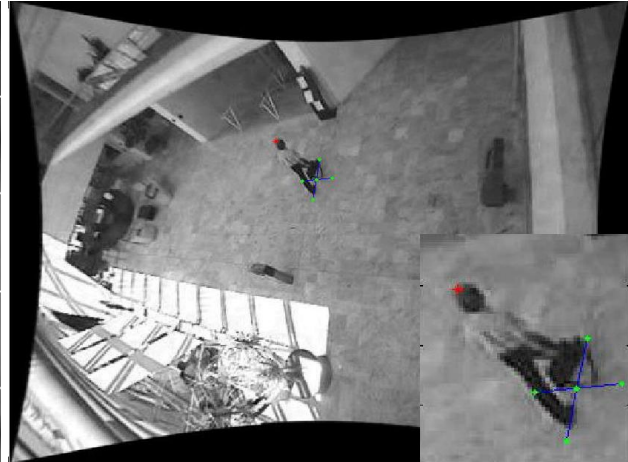

(b)

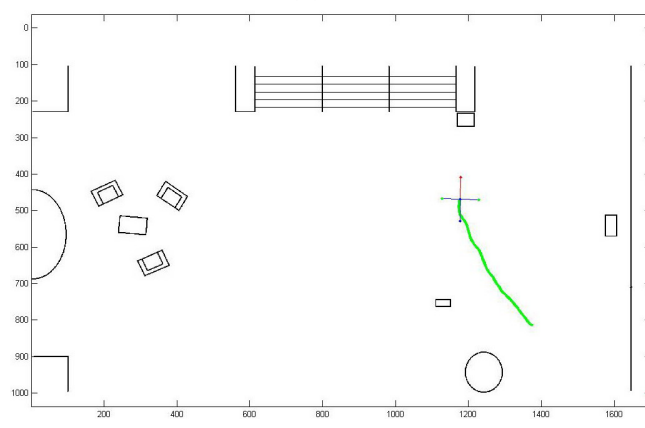

(c)

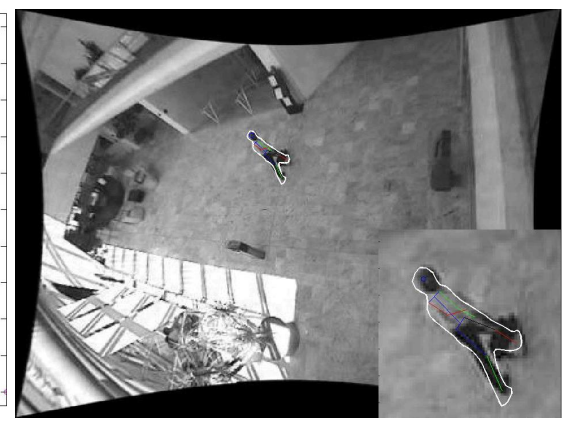

(d)

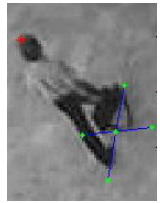

(e)

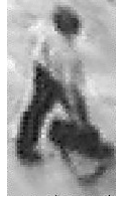

(f)

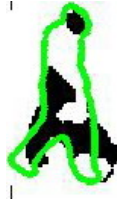

(g)

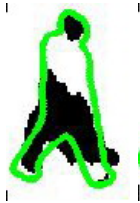

(h)

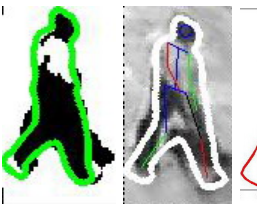

(i) (j)

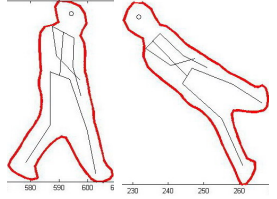

$(\mathrm{k})$
(1)

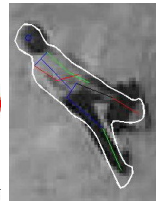

(m)

Figure 6: (a) Original input image LeftBagPickedUp0385. (b) Undistorted image $\mathbf{I}_{\mathbf{u n d i s t o r t e d ~}}$ with direction of motion. (c) Ground plane with trajectory and direction of motion. (d) Shape\&Skeleton plotted on $\mathbf{I}_{\mathbf{u n d i s t o r t e d}}$. (e) $\mathbf{I}_{\text {extracted }}$. (f) $I_{\text {projected }}$ ( $\left(\mathrm{g}, \mathrm{h}\right.$, i) Blob reconstruction. Shape \& Skeleton drawn on $I_{\text {projected }}$ (j), in frontal view $(\mathrm{k})$, in original image plane (1) and drawn on $\mathbf{I}_{\text {extracted }}(\mathrm{m})$.

In Fig.7, the result obtained for some frames of the Walk3 sequence can be observed. In the first phase of the sequence, the person is walking in a direction quite parallel to the image plane while in the second phase, he walks away: the "fronto-parallel" model is then used in the first case and the "fronto-orthogonal" one in the other. As shown in the $2^{\text {nd }}$ line of Fig.7.b and Fig.7.c, the result of the projection is surprisingly good: it seems that the camera is following the person, with the typical camera travelling effect in the "lateral" phase and with a backwards camera travelling track in the other phase. We can observe that a satisfactory scale factor is evaluated since the person presents a good height-width 
ratio in the warped image. This leads to a correct model fitting in that image ( $3^{r d}$ line). Resulting Shape and Skeleton are then back-projected to the original image ( $4^{\text {th }}$ line $)$.

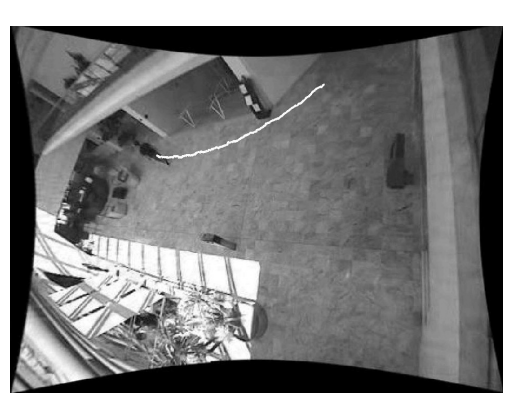

(a)

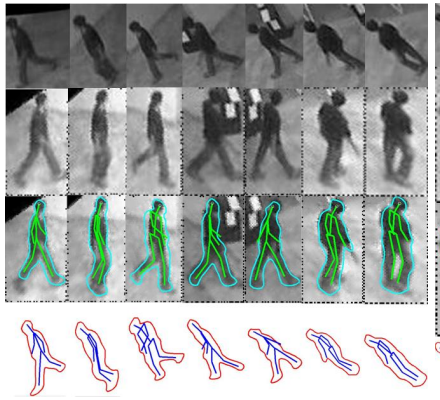

(b)

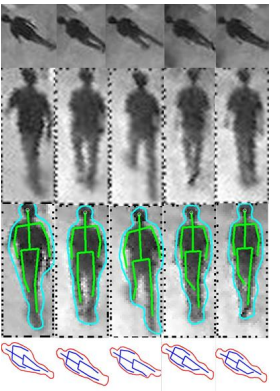

(c)

Figure 7: Walk3 sequence from frame 30500 to frame 30644: original image with trajectory (a), frontoparallel model application in the first phase (b) and fronto-orthogonal model application in the second phase (c). In (b) and (c), $1^{s t}$ line correspond to $\mathbf{I}_{\text {extracted }}, 2^{\text {nd }}$ line to $I_{\text {projected }} .3^{r d}$ line represents both Shape \& Skeleton drawn on $I_{\text {projected }}$ and $4^{\text {th }}$ line shows both Shape \& Skeleton drawn in original image plane.

Numerical results are presented in Fig.8. For each frame of the sequences, the proposed algorithm and the "classical" model are tested: the human figure is segmented and an evaluation is made of the stick figure. Firstly, we calculate the distance between the estimated head position and the manually labelled one. Then the ground position distance is also calculated in the same way, defining the ground position as the mean position of both feet. Compared with the method without projective transformation, the proposed one presents much better results: the error is less than 5 pixels, three times smaller than with the previous methodology. These preliminary results are very promising although further tests will have to be considered for a complete validation.

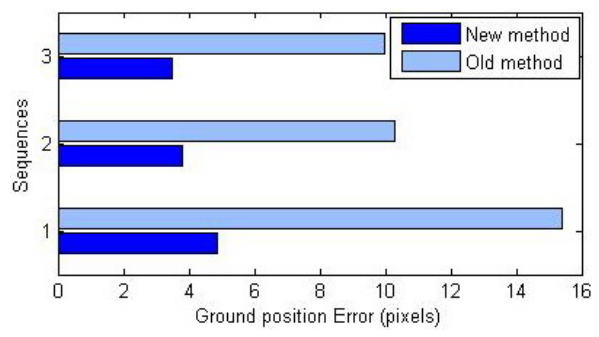

(a)

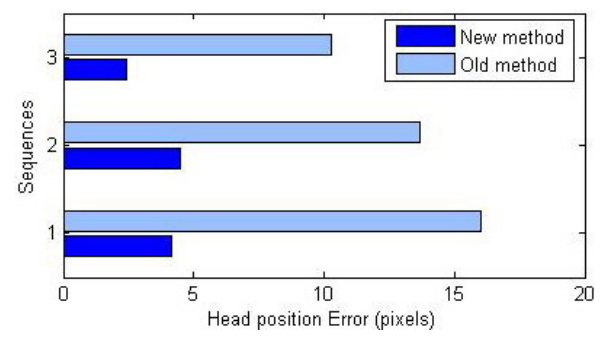

(b)

Figure 8: Numerical results obtained with Walk1, Walk2 and Walk3 sequences.

\section{Conclusions}

The proposed approach can be applied to every possible 2D view dependent models (shape, color or gradient based model). In this paper we have proposed a viewpoint independent PDM for human motion analysis. We have demonstrated that it is sufficient to model only two displacement angles with few intermediate models for obtaining 
interesting results. The presented model takes into account the position of the camera with respect to the person (since various training views were used) and the perspective effect: both training data base and original image to process are transformed to a frontal view, orthogonal or parallel to the direction of motion. Compared with the previous one, the proposed methodology presents much better results even if it has its limitations: the transformation is not always possible and the case of top-view is the extreme one.

In this work we only apply our model separately on each image without taking into account the motion dynamic. Future work relies on combining this approach with a particle filtering framework in order to obtain a robust human motion tracker in feature space and in the image: this would give a solution to the stationary case. Finally, more complicated cases such as various pedestrians with partial occlusions will be considered, and other kinds of motion should be taken into account in more complete models.

\section{References}

[1] EC funded CAVIAR project IST 200137540.

[2] R. Bowden, T. Mitchell, and M. Sarhadi. Non-linear statistical models for the 3d reconstruction of human pose and motion from monocular image sequences. Image Vision Comput., 18(9):729-737, 2000.

[3] P. Canotilho-Ribeiro and J. Santos-Victor. Human activity recognition from video: modeling, feature selection and classification architecture. HAREM, 2005.

[4] T.F. Cootes and C.J. Taylor. A mixture model for representing shape variation. British Machine Vision Association, pages 110-119, Essex, UK, 1997.

[5] A. Criminisi, I. D. Reid, and A. Zisserman. Single view metrology. International Journal of Computer Vision, 40(2):123-148, 2000.

[6] K. Grauman, G. Shakhnarovich, and T. Darrell. Example-based 3d shape inference from a single silhouettes. In ECCV Workshop SMVP, pages 1548-1553, 2004.

[7] R. Gross and J. Shi. The cmu motion of body (mobo) database, 2001.

[8] J.J. Guerrero and C. Sagüés. Robust line matching and estimate of homographies simultaneously. In IbPRIA, pages 297-307, 2003.

[9] Amit Kale, Amit K. Roy Chowdhury, and Rama Chellappa. Towards a view invariant gait recognition algorithm. In AVSS, pages 143-150, 2003.

[10] E. Lutton, H. Maitre, and J. Lopez-K. Contribution to the determination of vanishing points using hough transform. IEEE Trans. on PAMI, 16(4):430-438, 1994.

[11] P. Remagnino, A. Baumberg, T. Grove, D. Hogg, T. Tan, A. Worrall, and K. Baker. An integrated traffic and pedestrian model-based vision system. In BMVC, 1997.

[12] G. Rogez, C. Orrite, J. Martnez, and E. Herrero. Probabilistic spatio-temporal 2dmodel for pedestrian motion analysis in monocular sequences. In AMDO 2006.

[13] R. Rosales, M. Siddiqui, J. Alon, and S. Sclaroff. Estimating 3d body pose using uncalibrated cameras. In CVPR (1), pages 821-827, 2001. 